\begin{tabular}{|c|l|}
\hline Title & A uniqueness result on cauchy problem related to jump-ty pe markov processes with unbounded characteristics \\
\hline Author(s) & Mikami, Toshio \\
\hline Citation & $\begin{array}{l}\text { Stochastic A nalysis and A pplications, 20(2), 389-413 } \\
\text { https://doi.org/L0.1081/SAP-120003441 }\end{array}$ \\
\hline Issue Date & 2002 \\
\hline Doc URL & http://hdl.handle.net/2115/5926 \\
\hline Type & article (author version) \\
\hline Note(URL) & http://www.taylorandfrancisgroup.com/ \\
\hline File Information & SAA 20-2.pdf \\
\hline
\end{tabular}

Instructions for use 


\title{
A UNIQUENESS RESULT ON CAUCHY PROBLEM RELATED TO JUMP-TYPE MARKOV PROCESSES WITH UNBOUNDED CHARACTERISTICS
}

\author{
Toshio Mikami \\ Department of Mathematics, Hokkaido University, \\ Sapporo 060, Japan
}

\begin{abstract}
We give a sufficient condition for the uniqueness of solutions to Cauchy problem related to jump-type Markov processes with unbounded characteristics, by way of the exponential martingales and Chebychev's inequality. We also give an existence theorem for a class of functional-differential equations by a probabilistic method to show that our result is not meaningless.
\end{abstract}

\section{INTRODUCTION.}

Fix $T>0$ and $u_{0}(\cdot) \in C\left(\mathbf{R}^{n} ; \mathbf{R}\right)$, and consider the following PDE (see $[7,15])$; for $t \in(0, T)$ and $x \in \mathbf{R}^{n}$,

$$
\begin{aligned}
\partial u(t, x) / \partial t= & \sum_{i, j=1}^{n} a^{i j}(t, x) \partial^{2} u(t, x) / \partial x_{i} \partial x_{j} \\
& +\sum_{i=1}^{n} b^{i}(t, x) \partial u(t, x) / \partial x_{i}+f(t, x, u(t, x)), \\
u(0, x)= & u_{0}(x) .
\end{aligned}
$$

The result on the existence of solutions to (1.1) with $f \equiv 0$ can be found in [7, p. 142, Theorem 4.6] (see also section 4).

As a uniqueness theorem for (1.1), the following can be proved from [7, p. 140, Corollary 4.2]. 
Theorem 1.1. Suppose that $\left(a^{i j}(t, x)\right)_{i, j=1}^{n}$ is bounded and nonnegative definite, and that $f(t, x, u)$ is differentiable in $u$, and that the following holds;

$$
\begin{array}{r}
\sup \left\{(\partial f(t, x, u) / \partial u) /\left(1+|x|^{2}\right) ; t \in[0, T], x \in \mathbf{R}^{n}, u \in \mathbf{R}\right\}<\infty \\
\quad \sup \left\{\left|b^{i}(t, x)\right| /(1+|x|) ; 0 \leq t \leq T, x \in \mathbf{R}^{n}, i=1, \cdots, n\right\}<\infty
\end{array}
$$

Then any solutions $u_{1}(t, x)$ and $u_{2}(t, x) \in C^{1,2}\left((0, T) \times \mathbf{R}^{n} ; \mathbf{R}\right) \cap C([0, T] \times$ $\left.\mathbf{R}^{n} ; \mathbf{R}\right)$ to (1.1) coincide with each other if the following holds;

$$
\limsup _{x \rightarrow \infty}|x|^{-2} \log \left\{\sup _{0 \leq t \leq T}\left|u_{1}(t, x)-u_{2}(t, x)\right|+1\right\}<+\infty .
$$

Theorem 1.1 means that the uniqueness of solutions to (1.1) holds in the space $\left\{u(t, x) \in C\left([0, T] \times \mathbf{R}^{n} ; \mathbf{R}\right)\right.$; there exist $\alpha$ and $\beta>0$ such that $\sup _{0<t<T}|u(t, x)| \leq \alpha \exp \left(\beta|x|^{2}\right)$ for all $\left.x \in \mathbf{R}^{n}\right\}$.

The following result on the uniqueness of solutions to (1.1) is different from Theorem 1.1, and can be proved from [7, p. 141, Corollary 4.4].

Theorem 1.2. Suppose that $\left(a^{i j}(t, x)\right)_{i, j=1}^{n}$ is nonnegative definite, and that $f(t, x, u)$ is differentiable in $u$, and that the following holds;

$$
\begin{gathered}
\sup \left\{\partial f(t, x, u) / \partial u ; t \in[0, T], x \in \mathbf{R}^{n}, u \in \mathbf{R}\right\}<\infty, \\
\sup \left\{\left|a^{i j}(t, x)\right| /\left(1+|x|^{2}\right) ; 0 \leq t \leq T, x \in \mathbf{R}^{n}, i, j=1, \cdots, n\right\}<\infty, \\
\sup \left\{\left|b^{i}(t, x)\right| /(1+|x|) ; 0 \leq t \leq T, x \in \mathbf{R}^{n}, i=1, \cdots, n\right\}<\infty .
\end{gathered}
$$

Then any solutions $u_{1}(t, x)$ and $u_{2}(t, x) \in C^{1,2}\left((0, T) \times \mathbf{R}^{n} ; \mathbf{R}\right) \cap C([0, T] \times$ $\left.\mathbf{R}^{n} ; \mathbf{R}\right)$ to (1.1) coincide with each other if the following holds; there exists $q>0$ such that

$$
\limsup _{x \rightarrow \infty}\left(|x|^{-q} \sup _{0 \leq t \leq T}\left|u_{1}(t, x)-u_{2}(t, x)\right|\right)<+\infty .
$$

Theorem 1.2 means that the uniqueness result to (1.1) holds in the space $\left\{u(t, x) \in C\left([0, T] \times \mathbf{R}^{n} ; \mathbf{R}\right)\right.$; there exist $\alpha$ and $\beta>0$ such that $\sup _{0 \leq t \leq T}|u(t, x)| \leq \alpha\left(1+|x|^{\beta}\right)$ for all $\left.x \in \mathbf{R}^{n}\right\}$.

As an example of nonunique solutions to (1.1), the following is known (see also [14]). 
Theorem 1.3. ([1, Theorem]). Suppose that $n=1$ in (1.1) and that $a^{11}(t, x) \equiv 1, b^{1}(t, x) \equiv f(t, x, u) \equiv 0$. Then there exists a solution $u(t, x) \in$ $C^{1,2}((0, \infty) \times \mathbf{R} ; \mathbf{R}) \cap C([0, \infty) \times \mathbf{R} ; \mathbf{R})$ of $(1.1)$ with $T=\infty$ for which $u(0, x) \equiv 0$, and which is not identically zero for $t>0$, and for which for any $\varepsilon>0$, there exists a constant $C(\varepsilon)>0$ such that

$$
|u(t, x)| \leq C(\varepsilon) \exp (\varepsilon / t) \quad \text { on }(0, \infty) \times \mathbf{R} .
$$

From Theorem 1.1, for $u(t, x)$ in Theorem 1.3,

$$
\limsup _{x \rightarrow \infty}|x|^{-2} \log \left(\sup _{0 \leq t \leq T}|u(t, x)|+1\right)=\infty .
$$

But from the proof of Theorem 1.3, the following holds; there exist $C_{1}$ and $C_{2}>0$ such that for all $x \in \mathbf{R}^{n}$,

$$
\sup _{0 \leq t}|u(t, x)| \leq C_{1} \exp \left[C_{2} \exp \left(2 x^{2}\right)\right]
$$

(This can be shown by estimating $C_{M}$ in [1], and by putting $x=M-3 \varepsilon$ in [1].)

Remark 1.1. Theorem 1.3 implies that the smoothness of solutions and the nice property of coefficients of a differential operator in (1.1) do not imply the uniqueness of solutions to (1.1). What is important when we discuss the uniqueness of solutions to (1.1) is the space in which we consider (1.1). We refer the readers to [10] and the references therein for the uniqueness and non-uniqueness of positive weak solutions to (1.1) with $f \equiv 0$.

In this paper, we show that the space in which the uniqueness to (1.1) holds can be wider than that in Theorems 1.1-1.2, depending on the growth condition of $a(t, x) \equiv\left(a^{i j}(t, x)\right)_{i, j=1}^{n}$ and $b(t, x) \equiv\left(b^{i}(t, x)\right)_{i=1}^{n}$. In particular, there exists an example for which the set $\left\{u(t, x) \in C\left([0, T] \times \mathbf{R}^{n} ; \mathbf{R}\right)\right.$; there exist $\alpha$ and $\beta>0$ such that $\left.\sup _{0 \leq t \leq T}|u(t, x)| \leq \alpha \exp \left[\beta \exp \left(2 x^{2}\right)\right]\right\}$ is the set in which the uniqueness to (1.1) holds (see Example 2.1). We also discuss the generalizations of Theorems 1.1-1.2 (see Examples 2.2-2.3).

Considering the application to the optimal control of Markov processes, in this paper, we consider a class of nonlinear integro-differential equations (see $[2,16]$ and the references therein).

In section 2 we state our results. In section 3 we prove them. In section 4 we give a result on the existence of solutions to a class of nonlinear functional-differential equations to show that our result is not meaningless.

2. MAIN RESULT. 
Let us introduce our integro-differential equation.

Fix $T>0$ and $\phi(\cdot) \in C\left(\mathbf{R}^{n} ; \mathbf{R}\right)$, and consider the following integrodifferential equation; for $t \in(0, T)$ and $x \in \mathbf{R}^{n}$,

$$
\begin{aligned}
\partial u(t, x) / \partial t+\sum_{i, j=1}^{n} a^{i j}(t, x) \partial^{2} u(t, x) / \partial x_{i} \partial x_{j} & \\
+\sum_{i=1}^{n} b^{i}(t, x) \partial u(t, x) / \partial x_{i}+\int_{y \neq o, y \in \mathbf{R}^{n}}[u(t, y+x) & \\
\left.-u(t, x)-\sum_{i=1}^{n} y_{i} \partial u(t, x) / \partial x_{i}\right] \nu_{t, x}(d y) & =f(t, x, u(t, x)), \\
u(T, x) & =\phi(x)
\end{aligned}
$$

(see (A.1) below for the technical assumption on $a(t, x), b(t, x)$ and $\nu_{t, x}$ ).

In [16], they considered the case $a(t, x) \equiv 0$ and $f(t, x, u) \equiv 0$, and proved the uniqueness and the existence of bounded viscosity solution, under weaker assumption than ours (see Definition 2.1 and Theorem 4.3 in $[16])$.

In [2], they considered the case $a(t, x) \equiv 0$ and $f(t, x, u) \equiv 0$, under the assumption which is stronger than that in [16], but which is weaker than ours. They showed the uniqueness and the existence of a bounded classical solution to (2.1) (see Lemma 2.1 in [2]).

We point out that the uniqueness of bounded classical solutions to (2.1) with $f(t, x, u) \equiv 0$ can be proved by the Ito formula (see [9]), by using of the cut-off function, under the follwoing condition; for any $R>0$, there exists a Borel measure $\nu_{R}(d y)$ on $\mathbf{R}^{n} \backslash\{o\}$ such that $\nu_{R}(\{y ;|y|>1\})<\infty$ and $\nu_{t, x} \leq \nu_{R}$ for all $t, x$ for which $|x|<R, 0 \leq t \leq T$ (see (3.6)).

In this paper we discuss the uniqueness of unbounded classical solutions to (2.1). More precisely speaking, we find the space in which the uniqueness of (unbounded) classical solutions to (2.1) holds. The existence of solutions to $(2.1)$ will be discussed in section 4 .

We first state a technical assumption. (A.1). (1). $a(t, x)\left((t, x) \in[0, T] \times \mathbf{R}^{n}\right)$ is a symmetric $n \times n$-matrix, and is measurable, and is continuous in $x$, uniformly in $t \in[0, T]$. For any $R>0$, there exists $C>0$ such that

$$
|z|^{2} / C \leq<a(t, x) z, z>\leq C|z|^{2}
$$


for all $z \in \mathbf{R}^{n}, t \in[0, T]$, and $x \in \mathbf{R}^{n}$ for which $|x|<R$. Here $<\cdot, \cdot>$ denotes the inner product in $\mathbf{R}^{n}$. (2). $b(t, x)\left((t, x) \in[0, T] \times \mathbf{R}^{n}\right)$ is measurable and locally bounded. (3). $\nu_{t, x}(d y)\left((t, x) \in[0, T] \times \mathbf{R}^{n}\right)$ is a measurable, nonnegative Borel measure on $\mathbf{R}^{n} \backslash\{o\}$ such that the following holds;

$$
\sup _{t \in[0, T], x \in \mathbf{R}^{n}} \nu_{t, x}(y ;|y|>1)<\infty
$$

and for any $R>0$,

$$
\sup _{t \in[0, T],|x|<R} \int_{y \neq o} \min \left(|y|^{2},|y|\right) \nu_{t, x}(d y)<\infty .
$$

(4). There exists an increasing, concave function $k \in C^{1}([0, \infty) ;(0, \infty))$ such that

$$
\begin{gathered}
\int_{1}^{\infty}(y k(y))^{-1} d y=\infty \\
|<x, b(t, x)>|+\operatorname{Trace}[a(t, x)]+\int_{|y| \leq 1}|y|^{2} \nu_{t, x}(d y) \leq k(|x|)\left(1+|x|^{2}\right)
\end{gathered}
$$

for all $t \in[0, T]$ and $x \in \mathbf{R}^{n}$.

Remark 2.1. Under (A.1), for any $t \in[0, T]$ there exist strong Markov processes $\left(X(s), P_{t, x}\right)_{t \leq s \leq T, x \in \mathbf{R}^{n}}$ whose infinitesimal generator $L_{s}$ is given by the following; for any infinitely differentiable function $g ; \mathbf{R}^{n} \mapsto \mathbf{R}$ with a compact support, $s \in[t, T]$, and $x \in \mathbf{R}^{n}$,

$$
\begin{array}{r}
L_{s} g(x)=\sum_{i=1}^{n} b^{i}(s, x) \partial g(x) / \partial x_{i}+\sum_{i, j=1}^{n} a^{i j}(s, x) \partial^{2} g(x) / \partial x_{i} \partial x_{j} \\
\quad+\int_{y \neq o}\left[g(y+x)-g(x)-\sum_{i=1}^{n} y_{i} \partial g(x) / \partial x_{i}\right] \nu_{s, x}(d y) .
\end{array}
$$

Moreover

$$
X(s)=x+\int_{t}^{s} b(\alpha, X(\alpha)) d \alpha+M^{[c]}(s)+M^{[d]}(s) \quad \text { for } s \in[t, T]
$$


$P_{t, x^{-a . s .}}$, where $M^{[c]}(s)$ and $M^{[d]}(s)$ denote locally square integrable, continuous and purely discontinuous martingales, respectively (see [11], Theorems 2.1 and 5.2 and also $[12,17])$.

Next we state the assumptions related to the growth condition on the uniqueness of solutions to (2.1).

(A.2). There exist nonnegative functions $B$ and $H$ on $[0, \infty)$ such that for any $\tilde{r}>0$ and $r>0$

$$
\begin{aligned}
& B(\tilde{r}) H(r) \geq \sup \left\{\int_{y \neq o}[\exp (<z, y>)-1-<z, y>] \nu_{t, x}(d y)\right. \\
&+<a(t, x) z, z>; t \in[0, T],|x| \leq \tilde{r},|z| \leq r\}
\end{aligned}
$$

and such that

$$
\lim _{r \rightarrow \infty} r / B(r)=\infty
$$

There exists a constant $C(b)>0$ such that

$$
\sup \left\{|b(t, x)| /(1+|x|) ; t \in[0, T], x \in \mathbf{R}^{n}\right\}=C(b) .
$$

(A.3.1). There exists a continuous function $c(t, x)\left((t, x) \in[0, T] \times \mathbf{R}^{n}\right)$ such that $f(t, x, u)=-c(t, x) u$ and that

$$
C(c) \equiv \sup \left\{c(t, x) /(1+|x|) ;(t, x) \in[0, T] \times \mathbf{R}^{n}\right\}<\infty .
$$

(A.3.2). $f(t, x, u)\left((t, x, u) \in[0, T] \times \mathbf{R}^{n} \times \mathbf{R}\right)$ has a continuous $\partial f(t, x, u)$ $/ \partial u$, and

$$
\sup \left\{(\partial f(t, x, u) / \partial u) /(1+|x|) ; t \in[0, T], x \in \mathbf{R}^{n}, u \in \mathbf{R}\right\}<\infty .
$$

$(\mathrm{A} .2)^{\prime} \cdot \nu_{t, x}(d y) \equiv 0$. There exist a nondecreasing function $h$ and a smooth increasing function $g:[0, \infty) \mapsto(0, \infty)$, and positive constants $C_{1}(A)$, $C_{2}(A)$ and $C_{3}(A)$ such that

$$
\begin{aligned}
& C_{1}(A)=\sup \left\{g^{\prime}\left(|x|^{2}\right)<b(t, x), x>/ g\left(|x|^{2}\right) ; t \in[0, T], x \in \mathbf{R}^{n}\right\} \\
& C_{2}(A)=\sup \left\{\left[2 g^{\prime \prime}\left(|x|^{2}\right)<a(t, x) x, x>\right.\right. \\
& \left.\left.\quad+\operatorname{Trace}[a(t, x)] g^{\prime}\left(|x|^{2}\right)\right] / g\left(|x|^{2}\right) ; t \in[0, T], x \in \mathbf{R}^{n}\right\} \\
& C_{3}(A)=\sup \left\{\left|g^{\prime}\left(|x|^{2}\right)\right|^{2}<a(t, x) x, x>/ h\left(|x|^{2}\right)\right. \\
& \left.\quad ; t \in[0, T], x \in \mathbf{R}^{n}\right\} .
\end{aligned}
$$


(A.3.1)'. There exists a continuous function $c(t, x)\left((t, x) \in[0, T] \times \mathbf{R}^{n}\right)$ such that $f(t, x, u)=-c(t, x) u$ and that

$$
\begin{gathered}
\infty>\tilde{C}(c) \equiv \sup \{\sup \{c(t, x) ; t \in[0, T],|x| \leq r\} \\
\left./\left[1+g\left(r^{2}\right)^{2} / h\left(r^{2}\right)\right] ; r>0\right\} . \\
\lim _{r \rightarrow \infty} g(r)^{2} / h(r)=\infty .
\end{gathered}
$$

(A.3.2)'. (2.12) holds. $f(t, x, u)\left((t, x, u) \in[0, T] \times \mathbf{R}^{n} \times \mathbf{R}\right)$ has a continuous $\partial f(t, x, u) / \partial u$, and

$$
\begin{gathered}
\infty>\sup \{\sup \{(\partial f(t, x, u) / \partial u) ; t \in[0, T],|x| \leq r, u \in \mathbf{R}\} \\
\left./\left[1+g\left(r^{2}\right)^{2} / h\left(r^{2}\right)\right] ; r>0\right\} .
\end{gathered}
$$

The following is our main result.

Theorem 2.1. Suppose that (A.1), (A.2) and (A.3.1) hold. Then any solution $u(t, x) \in C^{1,2}\left((0, T) \times \mathbf{R}^{n} ; \mathbf{R}\right) \cap C\left([0, T] \times \mathbf{R}^{n} ; \mathbf{R}\right)$ to (2.1) with $u_{0}(x) \equiv 0$ is indentically zero if there exist positive constants $\alpha$ and $\beta$ such that

$$
\sup _{0 \leq t \leq T}|u(t, x)|<\alpha \exp (\beta|x|)
$$

for all $x \in \mathbf{R}^{n}$.

Theorem 2.2. Suppose that (A.1), (A.2)' and (A.3.1)' hold. Then any solution $u(t, x) \in C^{1,2}\left((0, T) \times \mathbf{R}^{n} ; \mathbf{R}\right) \cap C\left([0, T] \times \mathbf{R}^{n} ; \mathbf{R}\right)$ to $(2.1)$ with $u_{0}(x) \equiv 0$ is indentically zero if there exist positive constants $\alpha_{1}$ and $\beta_{1}$ such that

$$
\sup _{0 \leq t \leq T}|u(t, x)|<\alpha_{1} \exp \left[\beta_{1} g\left(|x|^{2}\right)^{2} / h\left(|x|^{2}\right)\right]
$$

for all $x \in \mathbf{R}^{n}$.

As corollaries to Theorems 2.1 and 2.2, we get the following. 
Corollary 2.1. Suppose that (A.1), (A.2) and (A.3.2) hold. Then any solutions $u_{1}(t, x)$ and $u_{2}(t, x) \in C^{1,2}\left((0, T) \times \mathbf{R}^{n} ; \mathbf{R}\right) \cap C\left([0, T] \times \mathbf{R}^{n} ; \mathbf{R}\right)$ to (2.1) coincide with each other if there exist positive constants $\alpha$ and $\beta$ such that

$$
\sup _{0 \leq t \leq T}\left|u_{1}(t, x)-u_{2}(t, x)\right|<\alpha \exp (\beta|x|)
$$

for all $x \in \mathbf{R}^{n}$.

Corollary 2.2. Suppose that (A.1), (A.2)'and (A.3.2)' hold. Then any solutions $u_{1}(t, x)$ and $u_{2}(t, x) \in C^{1,2}\left((0, T) \times \mathbf{R}^{n} ; \mathbf{R}\right) \cap C\left([0, T] \times \mathbf{R}^{n} ; \mathbf{R}\right)$ to (2.1) coincide with each other if there exist positive constants $\alpha_{1}$ and $\beta_{1}$ such that

$$
\sup _{0 \leq t \leq T}\left|u_{1}(t, x)-u_{2}(t, x)\right|<\alpha_{1} \exp \left[\beta_{1} g\left(|x|^{2}\right)^{2} / h\left(|x|^{2}\right)\right]
$$

for all $x \in \mathbf{R}^{n}$.

We close this section by the following examples. The first one is related to Theorem 1.3.

Example 2.1. Suppose that $\nu_{t, x}(d y)=c(t, x) \equiv 0$ and that the following holds;

$$
\sup \left\{\exp \left(3 x^{2}\right)\left(\sum_{i, j=1}^{n} a^{i j}(t, x)^{2}\right)^{1 / 2}+|x||b(t, x)| ; t \in[0, T], x \in \mathbf{R}^{n}\right\}<\infty
$$

Then (3) and (4) in (A.1) hold with $k(y)=\log (e+y)$, and (A.2)' and (2.12) hold with $g(r)=\exp (r)$ and $h(r)=1$, in which case

$$
g\left(|x|^{2}\right)^{2} / h\left(|x|^{2}\right)=\exp \left(2|x|^{2}\right) .
$$

If (1)-(2) in (A.1) holds, then from Theorem 2.2, the uniqueness of solutions to (1.1) with $f \equiv 0$ holds in the space $\left\{u(t, x) \in C\left([0, T] \times \mathbf{R}^{n} ; \mathbf{R}\right)\right.$; there exist $\alpha$ and $\beta>0$ such that $\sup _{0 \leq t \leq T}|u(t, x)| \leq \alpha \exp \left[\beta \exp \left(2 x^{2}\right)\right]$ for all $\left.x \in \mathbf{R}^{n}\right\}$ (see Theorem 1.3 and the following discussion).

Next we discuss the relation between Theorem 1.1 and Corollary 2.2. Example 2.2. Suppose that $a(t, x)$ is bounded and $\nu_{t, x}(d y) \equiv 0$, and that (1.2)-(1.3) holds. Then (3) and (4) in (A.1) hold with $k(y)=\log (e+y)$, and (A.2)' and (A.3.2)' hold with $g(r)=h(r)=r+1$, in which case 


$$
g\left(|x|^{2}\right)^{2} / h\left(|x|^{2}\right)=\left(|x|^{2}+1\right) .
$$

If (1)-(2) in (A.1) holds, then the uniqueness of solutions to (1.1) holds in the space $\left\{u(t, x) \in C\left([0, T] \times \mathbf{R}^{n} ; \mathbf{R}\right)\right.$; there exist $\alpha$ and $\beta>0$ such that $\sup _{0 \leq t \leq T}|u(t, x)| \leq \alpha \exp \left[\beta|x|^{2}\right]$ for all $\left.x \in \mathbf{R}^{n}\right\}$ from Corollary 2.2. This means that Corollary 2.2 gives the same growth condition on $a(t, x), b(t, x)$ and $\partial f(t, x, u) / \partial u$ to obtain the uniqueness of solutions to (1.1) as that in Theorem 1.1.

Finally we discuss the relation between Theorem 1.2 and Corollary 2.2. Example 2.3. Suppose that $\nu_{t, x}(d y) \equiv 0$ and that the following holds;

$$
\begin{aligned}
\infty>\sup \{ & \operatorname{Trace}[a(t, x)] /\left(1+|x|^{2}\{\log (e+|x|)\}\right) \\
& \left.+|b(t, x)| /(1+|x|\{\log (e+|x|)\}) ; t \in[0, T], x \in \mathbf{R}^{n}\right\}
\end{aligned}
$$

; and $f(t, x, u)$ has a continuous $\partial f(t, x, u) / \partial u$ for which

$$
\begin{gathered}
\infty>\sup \{\sup \{(\partial f(t, x, u) / \partial u) ; t \in[0, T],|x| \leq r, u \in \mathbf{R}\} \\
/(1+\log (r+e)) ; r>0\} .
\end{gathered}
$$

Then (3) and (4) in (A.1) hold with $k(y) \equiv \log (e+y)$, and (A.2)' and (A.3.2)' hold with $g(r)=\{\log (e+r)\}^{1 / 2}$ and $h(r)=1$, in which case

$$
\alpha_{1} \exp \left(\beta_{1} g\left(|x|^{2}\right)^{2} / h\left(|x|^{2}\right)\right)=\alpha_{1}\left(e+|x|^{2}\right)^{\beta_{1}}
$$

If (1)-(2) in (A.1) holds, then the uniqueness of solutions to (1.1) holds in the space $\left\{u(t, x) \in C\left([0, T] \times \mathbf{R}^{n} ; \mathbf{R}\right)\right.$; there exist $\alpha$ and $\beta>0$ such that $\sup _{0 \leq t \leq T}|u(t, x)| \leq \alpha\left(1+|x|^{\beta}\right)$ for all $\left.x \in \mathbf{R}^{n}\right\}$ from Corollary 2.2. This means that Corollary 2.2 gives the better growth condition on $a(t, x), b(t, x)$ and $\partial f(t, x, u) / \partial u$ to obtain the uniqueness of solutions to (1.1) than that in Theorem 1.2, that is, (2.21)-(2.22) is weaker than (1.5)-(1.6).

Examples 2.1-2.3 imply that the set in which the uniqueness of solutions to (1.1) holds becomes wider and wider as the growth order of $b(t, x)$ and $a(t, x)$, as $|x| \rightarrow \infty$, becomes smaller and smaller.

\section{PROOF OF RESULTS.}

In this section we prove Theorems in section 2 .

Before we prove them, let us give a notation and the technical result whose proof will be given later. 
For $r>0$, put

$$
\tau_{r} \equiv \inf \{t>0 ;|X(t)|>r\}
$$

The following lemma plays a crucial role in this paper.

Lemma 3.1. (I). Suppose that (A.1)-(A.2) hold. Then there exists a constant $t_{0} \in[0, T]$ such that for any $x \in \mathbf{R}^{n}$, and any $s, t$ for which $0 \leq t \leq s \leq \min \left(T, t+t_{0}\right)$,

$$
\lim _{r \rightarrow \infty} r^{-1} \log P_{t, x}\left(\tau_{r} \leq s\right)=-\infty .
$$

(II). Suppose that (A.1), (A.2)' and (2.12) hold. Then there exist constants $t_{0}$ and $C_{1}>0$ such that for any $x \in \mathbf{R}^{n}$, and any $s, t$ for which $0 \leq t \leq s \leq$ $\min \left(T, t+t_{0}\right)$

$$
\limsup _{r \rightarrow \infty} h\left(r^{2}\right) g\left(r^{2}\right)^{-2} \log P_{t, x}\left(\tau_{r} \leq s\right) \leq-C_{1} /(s-t) .
$$

Remark 3.1. $P_{t, x}\left(\tau_{r} \leq s\right)$ in Lemma 3.1 is the exit probability of $r^{-1} X(u)$ from the set $\left\{y \in \mathbf{R}^{n} ;|y|<1\right\}$. As $r \rightarrow \infty, r^{-1} X(u)$ converges to $o$. In this sense, $r^{-1} X(u)$ can be considered as the small random perturbations of $o$. The idea of the proof of Lemma 3.1 can be found in $[5,18,19]$. When $\nu_{t, x} \equiv 0$, then the method of viscosity solutions might be useful to prove (II) in Lemma 3.1. But when $\nu_{t, x} \not \equiv 0$, it can not be used (see Chap. 6 in [3]). In this case, the study of the asymptotic behavior of $r^{-1} X(u)$, as $r \rightarrow \infty$, is called the super large deviation (see [19], section 4.6), and it is not completely known.

Let us first prove Theorem 2.1 from Lemma 3.1, (I).

Proof of Theorem 2.1. Put

$$
C(c ; r) \equiv \sup \{c(t, y) ; t \in[0, T],|y| \leq r\}(\leq C(c)(1+r))
$$

(see $($ A.3.1)).

For $t \in[0, T], x \in \mathbf{R}^{n}$ and $r>|x|$,

$$
|u(t, x)| \leq E_{t, x}\left[\left|u\left(\tau_{r}, X\left(\tau_{r}\right)\right)\right| \exp \left(\int_{t}^{\tau_{r}} c(\gamma, X(\gamma)) d \gamma\right) ; T \geq \tau_{r}\right]
$$


which can be proved, by the Ito formula, from (A.2), (A.3.1) and (2.14). In fact, for $R>r$, take $\varphi_{R} \in C_{0}^{\infty}\left(\mathbf{R}^{n} ;[0,1]\right)$ for which $\varphi_{R}(y) \equiv 1$ for all $y(|y| \leq R)$. Then by applying the Ito formula (see $[9,12])$ to

$$
\begin{aligned}
& \varphi_{R}(X(s)) u(s, X(s)) \exp \left(\int_{t}^{s} c(\gamma, X(\gamma)) d \gamma\right) \quad\left(t \leq s \leq \min \left(T, \tau_{r}\right)\right) \\
& u(t, x) \\
& =E_{t, x}\left[\varphi_{R}\left(X\left(\tau_{r}\right)\right) u\left(\tau_{r}, X\left(\tau_{r}\right)\right) \exp \left(\int_{t}^{\tau_{r}} c(\gamma, X(\gamma)) d \gamma\right) ; T \geq \tau_{r}\right] \\
& \quad-E_{t, x}\left[\int_{t}^{\min \left(T, \tau_{r}\right)} \int_{y \neq o}\left(\varphi_{R}(y+X(s))-1\right)\right. \\
& \left.\quad \times u(s, y+X(s)) \nu_{s, X(s)}(d y) \exp \left(\int_{t}^{s} c(\gamma, X(\gamma)) d \gamma\right) d s\right]
\end{aligned}
$$

from (2.1). From (3.6), we get

$$
\begin{aligned}
& |u(t, x)| \\
& \leq E_{t, x}\left[\left|u\left(\tau_{r}, X\left(\tau_{r}\right)\right)\right| \exp \left(\int_{t}^{\tau_{r}} c(\gamma, X(\gamma)) d \gamma\right) ; T \geq \tau_{r}\right] \\
& \quad+E_{t, x}\left[\int_{t}^{\min \left(T, \tau_{r}\right)} \int_{|y+X(s)| \geq R}|u(s, y+X(s))| \nu_{s, X(s)}(d y)\right. \\
& \quad \times \exp ((s-t) C(c ; r)) d s],
\end{aligned}
$$

since $\varphi_{R} \in C_{0}^{\infty}\left(\mathbf{R}^{n} ;[0,1]\right)$. Let $R \rightarrow \infty$ in (3.7). Then we get (3.5), from (A.2), (3.4) and (2.14).

Hence, form (2.14), (3.4), (3.5) and Lemma 3.1, (I), we get, for $x \in \mathbf{R}^{n}$ and $t \in\left[T-t_{0}, T\right]$

$$
\begin{aligned}
& |u(t, x)| \\
& \leq \sum_{k=1}^{\infty} \sup \{|u(s, y)| ; t \leq s \leq T,|y| \leq(k+1) r\}
\end{aligned}
$$




$$
\begin{aligned}
& \quad \times \exp ((T-t) C(c ; r)) P_{t, x}\left(T \geq \tau_{r}, k r \leq\left|X\left(\tau_{r}\right)\right|<(k+1) r\right) \\
& \leq \sum_{k=1}^{\infty} \sup \{|u(s, y)| ; t \leq s \leq T,|y| \leq(k+1) r\} \\
& \quad \times \exp ((T-t) C(c ; r)) P_{t, x}\left(T \geq \tau_{k r}\right) \\
& \leq \sum_{k=1}^{\infty} \alpha \exp (\beta(k+1) r) \cdot \exp ((T-t) C(c)(1+r)) \\
& \quad \times \exp (-[3 \beta+C(c)(T-t)] k r)
\end{aligned}
$$

(for sufficiently large $r>0$, from Lemma 3.1, (I))

$=\alpha \exp ((T-t) C(c)-\beta r) /(1-\exp (-[2 \beta+C(c)(T-t)] r))$

$\rightarrow 0 \quad($ as $r \rightarrow \infty)$.

In the same way as in $(3.8), u(t, x)\left(\max \left(0, T-2 t_{0}\right) \leq t \leq T-t_{0}, x \in\right.$ $\mathbf{R}^{n}$ ) is identically zero. Inductively we can show that Theorem 2.1 is true.

Q.E.D.

Next we prove Theorem 2.2 from Lemma 3.1, (II).

Proof of Theorem 2.2. Take $t_{1} \in[0, T]$ sufficiently small so that

$$
\beta_{1}+t_{1} \tilde{C}(c)<C_{1} / t_{1} .
$$

From (3.5) and (A.3.1)', we get, for $x \in \mathbf{R}^{n}, r>|x|$ and $t \in\left[T-t_{1}, T\right]$

$$
\begin{aligned}
& |u(t, x)| \\
& \leq \sup _{t \leq s \leq T,|y|=r}|u(s, y)| \exp \left((T-t) \tilde{C}(c)\left[1+g\left(r^{2}\right)^{2} / h\left(r^{2}\right)\right]\right) P_{t, x}\left(T \geq \tau_{r}\right) \\
& \rightarrow 0 \quad(\text { as } r \rightarrow \infty \text { from Lemma } 3.1,(\mathrm{II}) \text { and }(2.15)) .
\end{aligned}
$$

Here we used the fact that the function $\beta_{1}+t \tilde{C}(c)-C_{1} / t$ of $t$ is increasing in $(0, \infty)$.

In the same way as above, $u(t, x)\left(\max \left(0, T-2 t_{1}\right) \leq t \leq T-t_{1}, x \in \mathbf{R}^{n}\right)$ is identically zero. Inductively we can show that Theorem 2.2 is true.

Q.E.D.

Before we prove Lemma 3.1, let us give some notation. For $r \in \mathbf{R}$, put

$$
L(r) \equiv \sup \{z r-H(z) ; z \geq 0\}
$$


(see $(2.3))$.

Remark 3.2. For $r>0$ and $z>0$,

$$
L(r) / r \geq z-H(z) / r .
$$

Let $r \rightarrow \infty$ and then $z \rightarrow \infty$. Then we get the following;

$$
\lim _{r \rightarrow \infty} L(r) / r=\infty .
$$

Finally we prove Lemma 3.1 .

Proof of Lemma 3.1. Let us first prove (I). For any $s, t$ such that $0 \leq t \leq$ $s \leq T, r>0$ and $x \in \mathbf{R}^{n}$,

$$
\begin{aligned}
P_{t, x}\left(\tau_{r} \leq s\right) & =P_{t, x}\left(\sup _{t \leq \gamma \leq s}|X(\gamma)| \geq r\right) \\
& \leq \sum_{i=1}^{n} P_{t, x}\left(\left|X\left(\tau_{r}\right)^{i}\right| \geq r / n, \tau_{r} \leq s\right) .
\end{aligned}
$$

For each $i=1, \cdots, n$ and any $R>0$,

$$
\begin{aligned}
& P_{t, x}\left(\left|X\left(\tau_{r}\right)^{i}\right| \geq r / n, \tau_{r} \leq s\right) \\
& \leq \exp (-r R / n) E_{t, x}\left[\exp \left(R X\left(\tau_{r}\right)^{i}\right)+\exp \left(-R X\left(\tau_{r}\right)^{i}\right) ; \tau_{r} \leq s\right]
\end{aligned}
$$

by the exponential Chebychev's inequality.

The right hand side of (3.15) can be considered as follows;

$$
\begin{aligned}
& E_{t, x}\left[\exp \left(R X\left(\tau_{r}\right)^{i}\right) ; \tau_{r} \leq s\right] \\
& =E_{t, x}\left[\operatorname { e x p } \left(R\left[X\left(\tau_{r}\right)^{i}-x_{i}\right]-\int_{t}^{\tau_{r}}\left\{R b^{i}(\gamma, X(\gamma))+a^{i i}(\gamma, X(\gamma)) R^{2}\right.\right.\right. \\
& \left.\quad+\int_{z \neq o}\left[\exp \left(R z_{i}\right)-1-R z_{i}\right] \nu_{\gamma, X(\gamma)}(d z)\right\} d \gamma \\
& \quad+R x_{i}+\int_{t}^{\tau_{r}}\left\{R b^{i}(\gamma, X(\gamma))+a^{i i}(\gamma, X(\gamma)) R^{2}\right. \\
& \left.\left.\left.\quad+\int_{z \neq o}\left[\exp \left(R z_{i}\right)-1-R z_{i}\right] \nu_{\gamma, X(\gamma)}(d z)\right\} d \gamma\right) ; \tau_{r} \leq s\right] \\
& \leq \exp [R|x|+(s-t)\{R C(b)(1+r)+B(r) H(R)\}]
\end{aligned}
$$


from (A.2), since

$$
\begin{aligned}
1=E_{t, x}[ & \exp \left(R\left[X\left(\min \left(\tau_{r}, s\right)\right)^{i}-x_{i}\right]\right. \\
& -\int_{t}^{\min \left(\tau_{r}, s\right)}\left\{R b^{i}(\gamma, X(\gamma))+a^{i i}(\gamma, X(\gamma)) R^{2}\right. \\
& \left.\left.\left.+\int_{z \neq o}\left[\exp \left(R z_{i}\right)-1-R z_{i}\right] \nu_{\gamma, X(\gamma)}(d z)\right\} d \gamma\right)\right]
\end{aligned}
$$

(see $[9,12])$. In the same way as in (3.16),

$$
\begin{aligned}
& E_{t, x}\left[\exp \left(-R X\left(\tau_{r}\right)^{i}\right) ; \tau_{r} \leq s\right] \\
& \leq \exp [R|x|+(s-t)\{R C(b)(1+r)+B(r) H(R)\}] .
\end{aligned}
$$

From (3.14)-(3.18), we get for $x(|x|<r)$,

$$
\begin{aligned}
& \log P_{t, x}\left(\tau_{r} \leq s\right) \\
& \leq \log (2 n)-(s-t)(R(r / n-|x|) /(s-t)-R C(b)(1+r)-B(r) H(R)) .
\end{aligned}
$$

Taking the infimum in $R>0$ on the left hand side of (3.19),

$$
\begin{aligned}
& \log P_{t, x}\left(\tau_{r} \leq s\right) \\
& \leq \log (2 n)-(s-t) B(r) \\
& \quad \times L\left(\left\{r\left[(n(s-t))^{-1}-C(b)\right]-|x| /(s-t)-C(b)\right\} / B(r)\right) .
\end{aligned}
$$

From (2.4), (3.20) and Remark 3.2,

$$
\limsup _{r \rightarrow \infty} r^{-1} \log P_{t, x}\left(\tau_{r} \leq s\right)=-\infty,
$$

provided that $[C(b) n]^{-1}>s-t$.

Let us prove (II). We prove the following; for any $s, t$ such that $0 \leq$ $t \leq s \leq T, x \in \mathbf{R}^{n}$ and $r>|x|$,

$$
\begin{aligned}
& P_{t, x}\left(\tau_{r} \leq s\right) \\
& \leq \exp \left(-g\left(r^{2}\right)^{2} /\left[(s-t) h\left(r^{2}\right)\right]\right. \\
& \left.\quad \times\left\{1-g\left(|x|^{2}\right) / g\left(r^{2}\right)-2(s-t)\left(C_{1}(A)+C_{2}(A)\right)\right\}^{2} /\left[16 C_{3}(A)\right]\right),
\end{aligned}
$$


provided that

$$
1>g\left(|x|^{2}\right) / g\left(r^{2}\right)+2(s-t)\left(C_{1}(A)+C_{2}(A)\right) .
$$

(3.23) holds for sufficiently large $r>0$ if $s-t<1 /\left[2\left(C_{1}(A)+C_{2}(A)\right)\right]$, since $g(r) \rightarrow \infty$ as $r \rightarrow \infty$ from (A.2)'.

Let us prove (3.22). We can show that for $C>0$

$$
\begin{aligned}
& P_{t, x}\left(\tau_{r} \leq s\right) \\
& =\exp \left(-C g\left(r^{2}\right)\right) E_{t, x}\left[\exp \left(C g\left(\left|X\left(\tau_{r}\right)\right|^{2}\right)\right) ; \tau_{r} \leq s\right] \\
& \leq \exp \left(-C g\left(r^{2}\right)\left[1-g\left(|x|^{2}\right) / g\left(r^{2}\right)-2(s-t)\left(C_{1}(A)+C_{2}(A)\right)\right]\right) \\
& \quad \times E_{t, x}\left[\exp \left(\int_{t}^{\tau_{r}} 2 C g^{\prime}\left(|X(\gamma)|^{2}\right)<X(\gamma), d M^{[c]}(\gamma)>\right) ; \tau_{r} \leq s\right] \\
& \leq \exp \left(-C g\left(r^{2}\right)\left[1-g\left(|x|^{2}\right) / g\left(r^{2}\right)-2(s-t)\left(C_{1}(A)+C_{2}(A)\right)\right]\right. \\
& \left.\quad+4(s-t) C^{2} h\left(r^{2}\right) C_{3}(A)\right)
\end{aligned}
$$

from (A.2)'.

Taking the infimum in $C>0$ in (3.24), we get (3.22).

Let us show that (3.24) is true. By the Ito formula (see $[9,12]), P_{t, x^{-}}$ a.s., if $\tau_{r} \leq s$,

$$
\begin{aligned}
& g\left(\left|X\left(\tau_{r}\right)\right|^{2}\right)-g\left(|x|^{2}\right) \\
& =\int_{t}^{\tau_{r}}\left[2 g^{\prime}\left(|X(\gamma)|^{2}\right)<X(\gamma), b(\gamma, X(\gamma))>\right. \\
& \quad+2 \operatorname{Trace}[a(\gamma, X(\gamma))] g^{\prime}\left(|X(\gamma)|^{2}\right) \\
& \left.\quad+4 g^{\prime \prime}\left(|X(\gamma)|^{2}\right)<a(\gamma, X(\gamma)) X(\gamma), X(\gamma)>\right] d \gamma \\
& \quad+\int_{t}^{\tau_{r}} 2 g^{\prime}\left(|X(\gamma)|^{2}\right)<X(\gamma), d M^{[c]}(\gamma)>
\end{aligned}
$$

(see $(2.2)$ ). Here we used the fact that $M^{[d]} \equiv 0$ if $\nu_{t, x} \equiv 0$ (see [12]).

We also have the following;

$$
E_{t, x}\left[\exp \left(\int_{t}^{\tau_{r}} 2 C g^{\prime}\left(|X(\gamma)|^{2}\right)<X(\gamma), d M^{[c]}(\gamma)>; \tau_{r} \leq s\right]\right.
$$




$$
\begin{aligned}
& =E_{t, x}\left[\operatorname { e x p } \left(\int_{t}^{\tau_{r}} 2 C g^{\prime}\left(|X(\gamma)|^{2}\right)<X(\gamma), d M^{[c]}(\gamma)>\right.\right. \\
& \quad-\int_{t}^{\tau_{r}} 4 C^{2}\left|g^{\prime}\left(|X(\gamma)|^{2}\right)\right|^{2}<a(\gamma, X(\gamma)) X(\gamma), X(\gamma)>d \gamma \\
& \left.\left.\quad+\int_{t}^{\tau_{r}} 4 C^{2}\left|g^{\prime}\left(|X(\gamma)|^{2}\right)\right|^{2}<a(\gamma, X(\gamma)) X(\gamma), X(\gamma)>d \gamma\right) ; \tau_{r} \leq s\right] \\
& \leq \exp \left(4(s-t) C^{2} h\left(r^{2}\right) C_{3}(A)\right)
\end{aligned}
$$

from (2.10), in the same way as in (3.16)-(3.17). Here we used the following; $M^{[c], i}(u) M^{[c], j}(u)-2 \int_{t}^{u} a^{i j}(\gamma, X(\gamma)) d \gamma$ is a local martingale.

From (3.25) and (3.26), we get (3.24).

Q.E.D.

\section{FUNCTIONAL-DIFFERENTIAL EQUATION.}

Since we couldn't find a reasonable result on the existence of classical solutions to $(2.1)$ with $a(t, x) \not \equiv 0$, we prove the existence of a bounded classical solution to (2.1). This will be done by considering the following functional differential equation; for $t \in(0, T)$ and $x \in \mathbf{R}^{n}$,

$$
\begin{aligned}
\partial v(t, x) / \partial t+\sum_{i, j=1}^{n} a^{i j}(t, x) \partial^{2} v(t, x) / \partial x_{i} \partial x_{j} & \\
+\sum_{i=1}^{n} B^{i}(t, x) \partial v(t, x) / \partial x_{i} & =F(v(t, \cdot), t, x), \\
v(T, x) & =\phi(x) .
\end{aligned}
$$

The assumption on $a(t, x)=\left(a^{i j}(t, x)\right)_{i, j=1}^{n}, B(t, x)=\left(B^{i}(t, x)\right)_{i=1}^{n}$, $F(\cdot, \cdot, \cdot)$ and $\phi(\cdot)$ will be stated later.

Remark 4.1. (4.1) formally becomes (2.1) if we put

$$
\begin{aligned}
& F(\varphi(\cdot), t, x) \\
& =-\int_{y \neq o, y \in \mathbf{R}^{n}}[\varphi(y+x)-\varphi(x)] \nu_{t, x}(d y)+f(t, x, \varphi(x)), \\
B(t, x) & =b(t, x)-\left(\int_{y \neq o, y \in \mathbf{R}^{n}} y_{i} \nu_{t, x}(d y)\right)_{i=1}^{n} .
\end{aligned}
$$


Let us state the assumption on the coefficients of the differential operator in (4.1).

(H.0). $a(t, x)=\sigma(t, x) \sigma(t, x)^{*} / 2$ is uniformly non-degenerate on $[0, T] \times$ $\mathbf{R}^{n}$. (Here $\sigma(t, x)=\left(\sigma^{i j}(t, x)\right)_{i, j=1}^{n}$ is a $n \times n$ matrix and $\sigma^{*}(t, x)$ denotes the transposed matrix of $\sigma(t, x)) \quad a.(t, x)$ and $B(t, x)$ are bounded and continuous. There exist constants $C(B)$ and $C(\sigma)>0$ such that for $t \in$ $[0, T]$ and $x^{\prime}, x \in \mathbf{R}^{n}$,

$$
\begin{aligned}
\left|B\left(t, x^{\prime}\right)-B(t, x)\right| & <C(B)\left|x^{\prime}-x\right|, \\
\sum_{i, j=1}^{n}\left|\sigma^{i j}\left(t, x^{\prime}\right)-\sigma^{i j}(t, x)\right|^{2} & <C(\sigma)\left|x^{\prime}-x\right|^{2} .
\end{aligned}
$$

From (H.0), there exist strong Markov processes $\{Y(s ; t, x)\}_{t \leq s \leq T}(0 \leq$ $t \leq T, x \in \mathbf{R}^{n}$ ) which satisfy the following stochastic differential equation; for $t \in[0, T], x \in \mathbf{R}^{n}$, and $s \in(t, T)$

$$
\begin{aligned}
d Y(s ; t, x) & =B(s, Y(s ; t, x)) d s+\sigma(s, Y(s ; t, x)) d W(s), \\
Y(t ; t, x) & =x,
\end{aligned}
$$

where $W(\cdot)$ is a n-dimensional Wiener process (see $[7,9]$ ).

Let us explain the idea to prove the existence of a classical solution to

We first need the following result; by the condition (H.1) below, from [7, p. 148, Theorem 5.3], one obtains Theorem 4.1.

(H.1). $h(t, x)\left((t, x) \in[0, T] \times \mathbf{R}^{n}\right)$ is bounded and continuous, and is Hölder continuous in $x$, uniformly with respect to $(t, x) \in[0, T] \times \mathbf{R}^{n} \cdot \phi(x)$ $\left(x \in \mathbf{R}^{n}\right)$ is bounded and continuous.

Theorem 4.1. Suppose that (H.0)-(H.1) hold. Then there exists a classical solution to the following $P D E$; for $t \in(0, T)$ and $x \in \mathbf{R}^{n}$,

$$
\begin{aligned}
\partial w(t, x) / \partial t+\sum_{i, j=1}^{n} a^{i j}(t, x) \partial^{2} w(t, x) / \partial x_{i} \partial x_{j} & \\
+\sum_{i=1}^{n} B^{i}(t, x) \partial w(t, x) / \partial x_{i} & =h(t, x), \\
w(T, x) & =\phi(x),
\end{aligned}
$$


and

$$
w(t, x)=E[\phi(Y(T ; t, x))]-\int_{t}^{T} E[h(s, Y(s ; t, x))] d s .
$$

To prove the existence of a bounded classical solution $v(t, x)$ to (4.1), we only have to prove the existence of a continuous solution to the following integral equation; for $t \in[0, T]$ and $x \in \mathbf{R}^{n}$,

$$
v(t, x)=E[\phi(Y(T ; t, x))]-\int_{t}^{T} E[F(v(s, \cdot), s, Y(s ; t, x))] d s
$$

such that the condition (H.1) is satisfied with $h(t, y)=F(v(t, \cdot), t, y)$.

In fact, if this is true, then (4.5) has a classical solution with $h(t, x)=$ $F(v(t, \cdot), t, x)$ from Theorem 4.1 and it is given by the right hand side of (4.6) with $h(t, x)=F(v(t, \cdot), t, x)$. This means that $v(t, x)$ in $(4.7)$ is a classical solution to (4.1).

Before we state the result on the existence of a classical solution to (4.1), let us give the assumption. We point out that $C_{b}\left([0, T] \times \mathbf{R}^{n} ; \mathbf{R}\right)$ and $C_{b}\left(\mathbf{R}^{n} ; \mathbf{R}\right)$ are given the topology by the uniform convergence in compact subsets of $[0, T] \times \mathbf{R}^{n}$ and of $\mathbf{R}^{n}$, respectively.

(H.2). $F(\cdot, \cdot, \cdot)$ is continuous in the following sense;

$$
\lim _{m \rightarrow \infty} F\left(\varphi_{m}, t_{m}, x_{m}\right)=F(\varphi, t, x)
$$

if $\left\{\varphi_{m}\right\}_{m \geq 1} \subset C_{b}\left(\mathbf{R}^{n} ; \mathbf{R}\right)$ are uniformly bounded and if $\left(\varphi_{m}, t_{m}, x_{m}\right)$ converges, as $m \rightarrow \infty$, to $(\varphi, t, x)$ in $C_{b}\left(\mathbf{R}^{n} ; \mathbf{R}\right) \times[0, T] \times \mathbf{R}^{n}$. There exist a positive nondecreasing function $C_{1}(r)(r \geq 0)$ and constants $C_{2}>0, \alpha_{1} \in(0,1]$ such that for any $r \geq 0, t \in[0, T], x, x^{\prime} \in \mathbf{R}^{n}$, and $\varphi, \varphi^{\prime} \in C_{b}\left(\mathbf{R}^{n} ; \mathbf{R}\right)$

$$
\left|F\left(\varphi, t, x^{\prime}\right)-F(\varphi, t, x)\right|<C_{1}(r)\left(\left|x^{\prime}-x\right|^{\alpha_{1}}+\sup _{\left|y^{\prime}-y\right| \leq\left|x^{\prime}-x\right|}\left|\varphi\left(y^{\prime}\right)-\varphi(y)\right|\right)
$$

as fas as $\sup _{y \in \mathbf{R}^{n}}|\varphi(y)| \leq r$; and

$$
\begin{aligned}
\left|F\left(\varphi^{\prime}, t, x\right)-F(\varphi, t, x)\right| & <C_{2} \sup _{y \in \mathbf{R}^{n}}\left|\varphi^{\prime}(y)-\varphi(y)\right|, \\
\sup \left\{|F(0, t, x)| ; t \in[0, T], x \in \mathbf{R}^{n}\right\} & <\infty .
\end{aligned}
$$


Here we denote by 0 the function of $x$ which is identically zero.

(H.3). $\phi(\cdot) \in C_{b}\left(\mathbf{R}^{n} ; \mathbf{R}\right)$. There exist constants $C_{3}>1$ and $\alpha_{2} \in(0,1]$ such that

$$
\left|\phi\left(x^{\prime}\right)-\phi(x)\right|<C_{3}\left|x^{\prime}-x\right|^{\alpha_{2}}
$$

for all $x^{\prime}, x \in \mathbf{R}^{n}$.

Under these conditions, we get the existence of a bounded classical solution to (4.1).

\section{Theorem 4.2.}

Suppose that (H.0) and (H.2)-(H.3) hold. Then (4.1) has a bounded classical solution $v(t, x)$ which is Hölder continuous in $x \in \mathbf{R}^{n}$, uniformly in $(t, x) \in[0, T] \times \mathbf{R}^{n}$.

Before we prove Theorem 4.2 , we state a result on the existence of a bounded classical solution to (2.1). Let us introduce the assumption.

(H.4). (H.0) holds with $B(t, x)=b(t, x)-\left(\int_{y \neq o} y_{i} \nu_{t, x}(d y)\right)_{i=1}^{n} \cdot \nu_{t, x}(d y)$ is weakly continuous in $(t, x) \in[0, T] \times \mathbf{R}^{n}$. There exists a finite Borel measure $\nu(d y)$ on $\mathbf{R}^{n} \backslash\{o\}$ such that $0 \leq \nu_{t, x} \leq \nu$ for all $(t, x) \in[0, T] \times \mathbf{R}^{n}$. There exist an increasing function $C_{4}:[0, \infty) \mapsto[0, \infty)$ and a constant $\beta_{1} \in(0,1]$ such that for any $h \in C_{b}\left(\mathbf{R}^{n} ; \mathbf{R}\right), x^{\prime}, x \in \mathbf{R}^{n}$ and $t \in[0, T]$,

$$
\left|\int_{y \neq o} h(y) \nu_{t, x^{\prime}}(d y)-\int_{y \neq o} h(y) \nu_{t, x}(d y)\right|<C_{4}\left(\sup _{y \in \mathbf{R}^{n}}|h(y)|\right)\left|x^{\prime}-x\right|^{\beta_{1}}
$$

$f(t, x, u)\left((t, x, u) \in[0, T] \times \mathbf{R}^{n} \times \mathbf{R}\right)$ is continuous. There exist constants $C_{5}>1$ and $\beta_{2} \in(0,1]$ such that

$$
\left|f\left(t, x^{\prime}, u^{\prime}\right)-f(t, x, u)\right|<C_{5}\left(\left|x^{\prime}-x\right|^{\beta_{2}}+\left|u^{\prime}-u\right|\right)
$$

for all $t \in[0, T], x^{\prime}, x \in \mathbf{R}^{n}$ and $u^{\prime}, u \in \mathbf{R}$.

$$
\sup \left\{|f(t, x, 0)| ; 0 \leq t \leq T, x \in \mathbf{R}^{n}\right\}<\infty .
$$

Under these conditions, we get the existence of a bounded classical solution to (2.1), directly from Theorem 4.2 and Remark 4.1, by Lebesgue's convergence theorem and the proof is omitted.

\section{Corollary 4.3.}

Suppose that (H.3)-(H.4) hold. Then (2.1) has a bounded classical solution which is Hölder continuous in $x \in \mathbf{R}^{n}$, uniformly in $(t, x) \in[0, T] \times \mathbf{R}^{n}$.

Before we prove Theorem 4.2, let us give a technical lemma whose proof is given for the sake of completeness. 
Lemma 4.1. Suppose that (H.0) holds. Then there exists a constant $C_{6}>$ 1 such that for any $s, t \in[0, T]$ with $t \leq s$ and $x^{\prime}, x \in \mathbf{R}^{n}$,

$$
E\left[\left|Y\left(s ; t, x^{\prime}\right)-Y(s ; t, x)\right|^{2}\right] \leq C_{6}\left|x^{\prime}-x\right|^{2} .
$$

(Proof). Applying the Ito formula (see $[7,9])$ to $\left|Y\left(s ; t, x^{\prime}\right)-Y(s ; t, x)\right|^{2}$, one gets

$$
\begin{aligned}
& E\left[\left|Y\left(s ; t, x^{\prime}\right)-Y(s ; t, x)\right|^{2}\right] \\
& =\left|x^{\prime}-x\right|^{2}+\int_{t}^{s} E\left[2<Y\left(u ; t, x^{\prime}\right)-Y(u ; t, x), B\left(u, Y\left(u ; t, x^{\prime}\right)\right)\right. \\
& \left.\quad-B(u, Y(u ; t, x))>+\sum_{i, j=1}^{n}\left|\sigma^{i j}\left(u, Y\left(u ; t, x^{\prime}\right)\right)-\sigma^{i j}(u, Y(u ; t, x))\right|^{2}\right] d s \\
& \leq\left|x^{\prime}-x\right|^{2}+[2 C(B)+C(\sigma)] \int_{t}^{s} E\left[\left|Y\left(u ; t, x^{\prime}\right)-Y(u ; t, x)\right|^{2}\right] d u
\end{aligned}
$$

from (H.0). By Gronwall's inequality (see [8]), one gets

$$
E\left[\left|Y\left(s ; t, x^{\prime}\right)-Y(s ; t, x)\right|^{2}\right] \leq\left|x^{\prime}-x\right|^{2} \exp ([2 C(B)+C(\sigma)](s-t)) .
$$

Let us prove Theorem 4.2.

Q.E.D.

(Proof of Theorem 4.2). Put $u_{0}(t, x) \equiv 0$. For $k \geq 1$, put inductively

$$
u_{k}(t, x)=E[\phi(Y(T ; t, x))]+\int_{t}^{T} E\left[F\left(u_{k-1}(s, \cdot), s, Y(s ; t, x)\right)\right] d s
$$
later.

The proof will be done by the following three steps which will be proved (Step I). For $k \geq 1, u_{k}(t, x)\left((t, x) \in[0, T] \times \mathbf{R}^{n}\right)$ is bounded and continuous. $\left\{u_{k}(t, x)\right\}_{k \geq 1,(t, x) \in[0, T] \times \mathbf{R}^{n}}$ is uniformly bounded. $u_{m}(t, x)$ converges, as $m \rightarrow \infty$, to a bounded continuous function $u_{\infty}(t, x)$, uniformly with respect to $t, x$ in compact subsets of $[0, T] \times \mathbf{R}^{n}$.

(Step II). There exist constants $C>1$ and $\alpha \in(0,1]$ such that for $k \geq 1$, $x^{\prime}, x \in \mathbf{R}^{n}$ with $\left|x^{\prime}-x\right|<1$ and $t \in[0, T]$, 


$$
\begin{aligned}
\left|u_{k}\left(t, x^{\prime}\right)-u_{k}(t, x)\right| & <\left\{C \sum_{i=0}^{k}(C(T-t))^{i} / i !\right\}\left|x^{\prime}-x\right|^{\alpha}, \\
\left|u_{\infty}\left(t, x^{\prime}\right)-u_{\infty}(t, x)\right| & <C \exp (C(T-t))\left|x^{\prime}-x\right|^{\alpha} .
\end{aligned}
$$

(Step III). $F\left(u_{\infty}(t, \cdot), t, x\right)\left((t, x) \in[0, T] \times \mathbf{R}^{n}\right)$ is bounded and continuous, and is Hölder continuous in $x$, uniformly with respect to $(t, x) \in[0, T] \times \mathbf{R}^{n}$.

Let us prove Theorem 4.2 from (Step I)-(Step III). By Lebesgue's convergence theorem, from the first part of (H.2) and (Step I), letting $k \rightarrow \infty$ in (4.16), one can show that $u_{\infty}(t, x)$ satisfies (4.7) with $v(t, x)=u_{\infty}(t, x)$. This is true, since in (4.16), for any $s \in[t, T]$,

$$
\lim _{k \rightarrow \infty}\left|F\left(u_{k-1}(s, \cdot), s, Y(s ; t, x)\right)-F\left(u_{\infty}(s, \cdot), s, Y(s ; t, x)\right)\right|=0
$$

from (H.2), and

$$
\begin{aligned}
& \left|F\left(u_{k-1}(s, \cdot), s, Y(s ; t, x)\right)-F\left(u_{\infty}(s, \cdot), s, Y(s ; t, x)\right)\right| \\
& \leq 2 C_{1} \sup _{0 \leq m,(t, x) \in[0, T] \times \mathbf{R}^{n}}\left|u_{m}(t, x)\right|
\end{aligned}
$$

from (4.9).

By Theorem 4.1, from (Step III), the proof is over (see the discussion below (4.7)).

Q.E.D.

Let us prove (Step I)-(Step III).

(Proof of (Step I)). We first show that $u_{k}(t, x)\left((t, x) \in[0, T] \times \mathbf{R}^{n}\right)$ is bounded and continuous for $k \geq 1$, by induction. $u_{0}(t, x) \equiv 0$ is clearly bounded and continuous. Suppose that $u_{k}(t, x)\left((t, x) \in[0, T] \times \mathbf{R}^{n}\right)$ is bounded and continuous. Then $F\left(u_{k}(t, \cdot), t, x\right)\left((t, x) \in[0, T] \times \mathbf{R}^{n}\right)$ is continuous from the first part of (H.2). Therefore we only have to show that $F\left(u_{k}(t, \cdot), t, x\right)\left((t, x) \in[0, T] \times \mathbf{R}^{n}\right)$ is bounded, to show that $u_{k+1}(t, x)$ $\left((t, x) \in[0, T] \times \mathbf{R}^{n}\right)$ is bounded and continuous (see (4.16)) since $Y(s ; t, x)$ is continuous in $(t, x) \in[0, T] \times \mathbf{R}^{n}$ for $s \in(t, T)$ almost surely (see [9]). This can be done as follows; for $(t, x) \in[0, T] \times \mathbf{R}^{n}$,

$$
\begin{aligned}
& \left|F\left(u_{k}(t, \cdot), t, x\right)\right| \\
& \leq|F(0, t, x)|+\left|F(0, t, x)-F\left(u_{k}(t, \cdot), t, x\right)\right| \\
& \leq \sup _{0 \leq s \leq T, y \in \mathbf{R}^{n}}\left(|F(0, s, y)|+C_{2}\left|u_{k}(s, y)\right|\right)<\infty
\end{aligned}
$$


from (4.9)-(4.10).

Next let us show that $\left\{u_{k}(t, x)\right\}_{k \geq 1,(t, x) \in[0, T] \times \mathbf{R}^{n}}$ is uniformly bounded, and that $u_{m}(t, x)$ converges, as $m \rightarrow \infty$, to a bounded continuous function $u_{\infty}(t, x)$, uniformly with respect to $t, x$ in compact subsets of $[0, T] \times \mathbf{R}^{n}$. For $k \geq 1$ and $t \in[0, T]$,

$$
\begin{aligned}
& \sup _{x \in \mathbf{R}^{n}}\left|u_{k+1}(t, x)-u_{k}(t, x)\right| \\
& \leq C_{2} \int_{t}^{T} \sup _{x \in \mathbf{R}^{n}}\left|u_{k}(s, x)-u_{k-1}(s, x)\right| d s
\end{aligned}
$$

from (4.9). Hence for $k \geq 1$ and $t \in[0, T]$,

$$
\begin{aligned}
& \sup _{x \in \mathbf{R}^{n}}\left|u_{k+1}(t, x)-u_{k}(t, x)\right| \\
& \leq\left\{\left(C_{2}\right)^{k}(T-t)^{k} / k !\right\} \sup _{0 \leq s \leq T, x \in \mathbf{R}^{n}}\left|u_{1}(s, x)-u_{0}(s, x)\right|
\end{aligned}
$$

which can be proved inductively.

From (4.21), $\left\{u_{k}(t, x)\right\}_{k \geq 0,(t, x) \in[0, T] \times \mathbf{R}^{n}}$ is uniformly bounded and converges to a bounded continuous function $u_{\infty}(t, x)$, uniformly with respect to $t, x$ in compact subsets of $[0, T] \times \mathbf{R}^{n}$ (see $[8]$ ).

Q.E.D.

(Proof of (Step II)). Put

$$
C \equiv\left[C_{1}\left(\sup _{0 \leq k, 0 \leq t \leq T, x \in \mathbf{R}^{n}}\left|u_{k}(t, x)\right|\right)+C_{3}\right] C_{6}+2 \sup _{0 \leq k, 0 \leq t \leq T, x \in \mathbf{R}^{n}}\left|u_{k}(t, x)\right|
$$

which is finite from (Step I) and (H.2), and put

$$
\alpha \equiv \min \left(\alpha_{1}, \alpha_{2}\right)
$$

We show that (4.17) is true for these $C$ and $\alpha$, inductively. (4.17) is true when $k=0$. Suppose that (4.17) is true for $k(\geq 1)$. Then for $x^{\prime}, x \in \mathbf{R}^{n}$ and $t \in[0, T]$ 


$$
\begin{aligned}
& \left|u_{k+1}\left(t, x^{\prime}\right)-u_{k+1}(t, x)\right| \\
& \leq C_{3} E\left[\left|Y\left(T ; t, x^{\prime}\right)-Y(T ; t, x)\right|^{\alpha_{2}}\right] \\
& \quad+C_{1}\left(\sup _{0 \leq m, 0 \leq s \leq T, y \in \mathbf{R}^{n}}\left|u_{m}(s, y)\right|\right) \int_{t}^{T} E\left[\left|Y\left(s ; t, x^{\prime}\right)-Y(s ; t, x)\right|^{\alpha_{1}}\right. \\
& \left.\quad+\left\{C \sum_{i=0}^{k}(C(T-s))^{i} / i !\right\}\left|Y\left(s ; t, x^{\prime}\right)-Y(s ; t, x)\right|^{\alpha}\right] d s
\end{aligned}
$$

from (4.8), (H.3) and the assumption on induction. This is true, since

$$
\begin{aligned}
& u_{k+1}\left(t, x^{\prime}\right)-u_{k+1}(t, x) \\
& =E\left[\phi\left(Y\left(T ; t, x^{\prime}\right)\right)\right]-E[\phi(Y(T ; t, x))] \\
& \quad+\int_{t}^{T} E\left[F\left(u_{k}(s, \cdot), s, Y\left(s ; t, x^{\prime}\right)\right)-F\left(u_{k}(s, \cdot), s, Y(s ; t, x)\right)\right] d s
\end{aligned}
$$

from (4.16), and since

$$
\begin{aligned}
& \left|F\left(u_{k}(s, \cdot), s, Y\left(s ; t, x^{\prime}\right)\right)-F\left(u_{k}(s, \cdot), s, Y(s ; t, x)\right)\right| \\
& \leq C_{1}\left(\sup _{0 \leq m, 0 \leq s \leq T, y \in \mathbf{R}^{n}}\left|u_{m}(s, y)\right|\right)\left\{\left|Y\left(s ; t, x^{\prime}\right)-Y(s ; t, x)\right|^{\alpha_{1}}\right. \\
& \left.\quad+\sup _{\left|y^{\prime}-y\right| \leq\left|Y\left(s ; t, x^{\prime}\right)-Y(s ; t, x)\right|}\left|u_{k}\left(s, y^{\prime}\right)-u_{k}(s, y)\right|\right\}
\end{aligned}
$$

from (4.8), and since

$$
\begin{aligned}
& \sup \left\{\left|u_{k}\left(s, y^{\prime}\right)-u_{k}(s, y)\right| ;\left|y^{\prime}-y\right| \leq\left|Y\left(s ; t, x^{\prime}\right)-Y(s ; t, x)\right|\right\} \\
& \leq\left\{\begin{array}{cl}
C \sum_{i=0}^{k}(C(T-s))^{i} / i !\left|Y\left(s ; t, x^{\prime}\right)-Y(s ; t, x)\right|^{\alpha} & \\
\operatorname{if}\left|Y\left(s ; t, x^{\prime}\right)-Y(s ; t, x)\right|<1, & \text { otherwise } \\
2 \sup _{0 \leq m, 0 \leq s \leq T, y \in \mathbf{R}^{n}}\left|u_{m}(s, y)\right|<C &
\end{array}\right.
\end{aligned}
$$

from the assumption on induction and (4.22), and

$$
C<C \sum_{i=0}^{k}(C(T-s))^{i} / i !\left|Y\left(s ; t, x^{\prime}\right)-Y(s ; t, x)\right|^{\alpha}
$$


if $\left|Y\left(s ; t, x^{\prime}\right)-Y(s ; t, x)\right| \geq 1$.

From (4.24) and Lemma 4.1, we get by Hölder's inequality,

$$
\begin{aligned}
& \left|u_{k+1}\left(t, x^{\prime}\right)-u_{k+1}(t, x)\right| \\
& \leq C_{3} C_{6}\left|x^{\prime}-x\right|^{\alpha_{2}}+C_{1}\left(\sup _{0 \leq m, 0 \leq s \leq T, y \in \mathbf{R}^{n}}\left|u_{m}(s, y)\right|\right) C_{6} \\
& \times\left[(T-t)\left|x^{\prime}-x\right|^{\alpha_{1}}+\left|x^{\prime}-x\right|^{\alpha} \int_{t}^{T}\left\{C \sum_{i=0}^{k}(C(T-s))^{i} / i !\right\} d s\right] \\
& \leq C\left\{\sum_{i=0}^{k+1}(C(T-t))^{i} / i !\right\}\left|x^{\prime}-x\right|^{\alpha}
\end{aligned}
$$

provided that $\left|x^{\prime}-x\right|<1$ from (4.23). Here we used the follows; $C_{6}>1$, and from (4.22),

$$
\begin{gathered}
C_{3} C_{6}<C, \\
C_{1}\left(\sup _{0 \leq m, 0 \leq s \leq T, y \in \mathbf{R}^{n}}\left|u_{m}(s, y)\right|\right) C_{6}[1+C] \\
=C C_{6}\left[C_{1}\left(\sup _{0 \leq m, 0 \leq s \leq T, y \in \mathbf{R}^{n}}\left|u_{m}(s, y)\right|\right)\right. \\
\left.+C_{1}\left(\sup _{0 \leq m, 0 \leq s \leq T, y \in \mathbf{R}^{n}}\left|u_{m}(s, y)\right|\right) / C\right]<C^{2},
\end{gathered}
$$

since

$$
\begin{aligned}
& C_{1}\left(\sup _{0 \leq m, 0 \leq s \leq T, y \in \mathbf{R}^{n}}\left|u_{m}(s, y)\right|\right) / C \\
& <C_{1}\left(\sup _{0 \leq m, 0 \leq s \leq T, y \in \mathbf{R}^{n}}\left|u_{m}(s, y)\right|\right) C_{6} / C<1<C_{3}
\end{aligned}
$$

(see (H.3)).

Letting $k \rightarrow \infty$ in (4.25), we get (4.18).

Q.E.D.

(Proof of (Step III)). Since $u_{\infty}(t, x)$ is bounded and continuous from (Step I), so is $F\left(u_{\infty}(t, \cdot), t, x\right)$ from (H.2) (see (4.19)).

Let us show that $F\left(u_{\infty}(t, \cdot), t, x\right)$ is Hölder continuous in $x$, uniformly with respect to $(t, x) \in[0, T] \times \mathbf{R}^{n}$.

From (4.8), for $t \in[0, T]$ and $x^{\prime}, x \in \mathbf{R}^{n}$, with $\left|x^{\prime}-x\right|<1$, 


$$
\begin{aligned}
& \left|F\left(u_{\infty}(t, \cdot), t, x^{\prime}\right)-F\left(u_{\infty}(t, \cdot), t, x\right)\right| \\
& \leq C_{1}\left(\sup _{(s, y) \in[0, T] \times \mathbf{R}^{n}}\left|u_{\infty}(s, y)\right|\right)\left\{\left|x^{\prime}-x\right|^{\alpha_{1}}\right. \\
& \left.\quad+\sup _{\left|y^{\prime}-y\right| \leq\left|x^{\prime}-x\right|}\left|u_{\infty}\left(t, y^{\prime}\right)-u_{\infty}(t, y)\right|\right\} \\
& \quad \leq C_{1}\left(\sup _{(s, y) \in[0, T] \times \mathbf{R}^{n}}\left|u_{\infty}(s, y)\right|\right)\{1+C \exp (C(T-t))\}\left|x^{\prime}-x\right|^{\alpha}
\end{aligned}
$$

from (4.18) and (4.23).

\section{ACKNOWLEDGEMENT.}

Q.E.D.

The author would like to thank Prof. M. Takeda for the useful suggestion.

\section{REFERENCES}

[1] S. Y. Chung and D. Kim: An example of nonuniqueness of the Cauchy problem for the heat equation. Comm. P.D.E. 19 (1994), 1257-1261.

[2] W. H. Fleming and H. M. Soner: Asymptotic expansions for Markov processes with Lévy generator. Appl. Math. Optim. 19 (1989), 203223.

[3] W. H. Fleming and H. M. Soner: Controlled Markov processes and Viscosity solutions. Springer-Verlag, Berlin, Heidelberg, New York, Tokyo, 1993.

[4] M. I. Freidlin: Random perturbations of reaction-diffusion equations: the quasi-deterministic approximation. Trans. Amer. Math. Soc. $\underline{305}$ (1988), 665-697.

[5] M. I. Freidlin and A. D. Wentzell: Random perturbations of dynamical systems. Springer-Verlag, Berlin, Heidelberg, New York, Tokyo, 1984.

[6] A. Friedman: Partial differential equations of parabolic type. Prentice - Hall, Englewood Cliffs, N.J., 1964.

[7] A. Friedman: Stochastic differential equations and applications, Vol. 1. Academic press, New York, 1975. 
[8] P. Hartman: Ordinary differential equations. John Wiley \& Sons, Inc., New York, London, Sydney, 1964.

[9] N. Ikeda and S. Watanabe: Stochastic differential equations and diffusion processes, 2nd ed. North-Holland/Kodansha, Amsterdam, New York, Oxford, Tokyo, 1989.

[10] K. Ishige and M. Murata: An intrinsic metric approach to uniqueness of the positive Cauchy problem for parabplic equations. TITECHMATH $11-95(\# 52)$ (1995).

[11] T. Komatsu: Markov processes associated with certain integro - differential equations. Osaka J. Math. $\underline{10}$ (1973), 271-303.

[12] P. A. Meyer: Un cours sur les intégrale stochastiques. In Meyer, P. A.(ed), Séminaire de Probabilités X, Université de Strasbourg 1974/75, Lecture Notes in Math. $\underline{511}$ (1976) 245-400, Springer-Verlag, Berlin, Heidelberg, New York, Tokyo.

[13] T. Mikami: Some generalizations of Wentzell's lower estimates on large deviations. Stochastics 24 (1988), 269-284.

[14] J. Rauch: Partial differential equations. Springer-Verlag, Berlin, Heidelberg, New York, Tokyo, 1991.

[15] J. Smoller: Shock waves and reaction-diffusion equations. SpringerVerlag, Berlin, Heidelberg, New York, Tokyo, 1983.

[16] H. M. Soner: Optimal control of jump Markov processes and viscosity solutions. In W. H. Fleming and P. L. Lions (eds), Proceedings of the IMA, University of Minnesota, June 1986, IMA Vols in Math. and Appl. 10 (1988) 501-511, Springer-Verlag, Berlin, Heidelberg, New York, Tokyo.

[17] D. W. Stroock: Diffusion processes with Lévy Generators. Zeit. Wahrsch. Verw. Gegiete $\underline{32}$ (1975), 209-244.

[18] A. D. Wentzell: Rough limit theorems on large deviations for Markov stochastic processes III. Theory Probab. Appl. 24 (1979), 675-692.

[19] A. D. Wentzell: Limit theorems on large deviations for Markov stochastic processes. Kluwer Academic Publishers, Dordrecht, 1990. 